\title{
TV-Medizinsendungen und Medizingeschichte - ein Dosis- und Darreichungsproblem auf dem deutschen Fernsehmarkt?
}

\author{
Christian Floto
}

Der Beitrag wendet sich den Fragen zu, inwieweit im deutschen TV-Programmangebot medizingeschichtliche Aspekte überhaupt thematisiert werden, inwiefern dieses im Zusammenhang mit Medizin bezogenen bzw. auch anderen Sendungen erfolgt, und in welchen Programmformen dieses geschieht. Dabei zeigt ein Vergleich der angloamerikanischen Programmofferten mit den biesigen TV-Spielplänen, dass dort eine bemerkenswerte Opulenz und Angebotsdifferenzierung zu Wissenschaftsthemen vorliegen, die hier ibresgleichen suchen. Zudem dominiert im deutschen Fernsebangebot in der Darstellung von Medizinthemen die Magazinform, wäbrend beispielsweise auf dem angloamerikanischen TV-Markt viele dieser Topoi-eben auch medizinhistorische - in "Langform" als Dokumentationen realisiert, gesendet und vertrieben werden. Als Gründe für diese Unterschiedlichkeit werden u. a. Programmtraditionen, "gelernte Rezeption", unterschiedliche Wertschätzung von Dokumentationen, programmplanerische Aspekte und Kostengesichtspunkte angefübrt. Zudem wird auf produzentenseitige Defizite in Wechselbeziebung mit tradierter Vernachlässigung bzw. Verkürzung medizinhistorischer Zusammenhänge verwiesen. Allerdings ist prognostisch aufgrund von Contentbedarf für Sendeplätze, Output- bzw. Ankaufpakete-Deals und unabhängigen Lizenzinhabern bei privaten Sendeveranstaltern mit einer internationalen Relativierung der bisher beobachtbaren Unterschiede zu rechnen.

Keywords: Medizingeschichte, Wissenschaftsthemen im Fernsehen, Wissenschaftsmagazine, Wissenschaftsdokumentationen, Programmplanung

\section{Einleitung}

Medizin und Geschichte gehen in der akademischen Fachdisziplin der „Medizingeschichte“ eine analytisch-retrospektivisch begründete Betrachtungsallianz ein, die personale Leistungen wie epochale Entwicklungen, Ereignisse, Entdeckungen, Handlungen und Verhaltensweisen unterschiedlicher Systemakteure im jeweiligen Politik- und Gesellschaftskontext aufzuzeigen und zu verstehen versucht. Dabei geht es $u$. a. auch darum, den tatsächlichen Anteil eines/einer Einzelnen an einem medizinhistorischen Erkenntnisgewinn zu ermitteln ${ }^{1}$. Die Bedeutung einer solchen Klärung mag etwa am Beispiel der lange Zeit strittigen Erst-Entdeckung des so genannten AIDS-Erregers HIV

1 Kommt es allerdings zu einer filmischen Dokumentation i. S. der Nutzung des Portraits als Darstellungsform der Vermittlung des Historischen, verweist Bleicher (1999, S. 174 ) auf Nichols: „Sobald eine lebende Person als ewiges Symbol mythisch komplettiert wird, verliert sich ihre provisorische Position innerhalb des historischen Feldes, (...). Das Historische erstarrt im Mythischen." 
durch den Franzosen Luc Montagnier - und eben nicht durch den Amerikaner Robert Gallo - deutlich werden.

Im Folgenden soll nun die Frage näher untersucht werden, inwieweit medizingeschichtliche Aspekte überhaupt im bzw. für das Fernsehen thematisiert werden, und inwiefern dieses im Zusammenhang mit medizinbezogenen bzw. auch anderen Sendungen im deutschen Fernsehen erfolgt - und in welchen Programmformen dieses geschieht. Denn für die narrative Darstellung dieser Inhalte kommt idealiter nicht zwangsläufig nur Dokumentarfilm bzw. Dokumentation/Feature in Betracht, auch wenn allein aufgrund ihrer üblichen Mindestlänge solche Formate ${ }^{2}$ eine hinreichende zeitliche Breite für die meist angestrebte inhaltliche Vertiefung bieten, die bei anderen Formen (z. B. Magazinbeitrag) nicht zur Verfügung steht ${ }^{3}$. Diese auf den ersten Blick einfach anmutende Fragestellung impliziert jedoch im Lichte der Programmwirklichkeit tatsächlich eine Reihe weiterer Aspekte, deren erschöpfende Klärung in diesem Zusammenhang nicht möglich ist, die aufzuwerfen allerdings die Bearbeitungslogik des eigentlichen Themas erfordert. Im Einzelnen handelt es sich um folgende Punkte bzw. Fragestellungen:

- Welche Sendungen sind - rezipientenseitig als solche wahrgenommen - überhaupt spezifische „Medizinsendungen“?

- Gibt es einen Unterschied zwischen „Gesundheits“- und „Medizin“-Sendungen?

- Welchen (und wie viel?) Medizin- und/oder Gesundheits-Bezug haben so genannte Wissenschaftssendungen? Was sind Wissenschaftssendungen, und in welchen Programmformen und -formaten finden sie sich im deutschen Fernsehen ${ }^{4}$ ?

- Welche Programmform erscheint für welchen medizinhistorischen Topos geeignet oder denkbar? Welche findet sich hiervon tatsächlich im deutschen Fernsehprogramm?

- Wer trifft Entscheidungen über „Fernseh-Spielpläne“ und Produktionen (eben auch zu medizinhistorischen Themen) und wie werden sie getroffen (z. B. Gremien- oder Einzelentscheidungen)?

- In welchem Beziehungs-, Entscheidungs- und möglichen Spannungsverhältnis stehen Entscheider und Produzenten zueinander?

2 In Deutschland Nettolängen von ca. 28'30" und 42'30"; im Gegensatz zum sonstigen internationalen Markt, der Längen von ca. 30' und 50'-55' standardisiert hat.

3 Leiser (1992) etwa bestätigt grundsätzlich die Eignung von Dokumentarfilmen zur Darstellung von Geschichte: „Wenn es darum geht zu erfahren, ,wie es wirklich gewesen ist’, ist der Film ohne Zweifel ein wichtiges und nützliches Hilfsmittel“. Zugleich mahnt er aber auch die kritische Würdigung von (nicht selbst gedrehtem) Material an.

4 Auf den ersten Blick scheinen Magazine (z. B. „Globus“, ARD; „Abenteuer Forschung“, ZDF; „Galileo“, Pro7; Formate in den Dritten Programmen wie „Quarks und Co.“, WDR; oder „Prisma“, NDR) von monatlicher bis werktäglicher (Pro7) Frequenz zu dominieren. Betrachtet man allerdings einen Sendeplatz wie den Termin am Sonntag um 19.30 Uhr im ZDF, ist auch dieser als Programmslot für Wissenschaftssendungen - hier: Dokumentationen! - deutbar. Ähnlich verhält es sich mit anderen Dokumentations-Sendeplätzen (auch bei privaten Fernsehveranstaltern), die zumindest teilweise mit Wissenschaftsthemen besetzt werden. Die Zuordnung mutet auch in der offiziellen Programmstatistik (Krüger 2002) beliebig an: Dort wird nicht näher zwischen Kultur-, Wissenschafts- und Ratgebersendungen im Einzelnen unterschieden; so wird z. B. die nah am jeweiligen wissenschaftlichen Fortschritt ausgerichtete Medizin-(und Gesundheits-!) Sendung „Praxis“ (ZDF) nicht dem Wissenschaftssendungs-Kontingent, sondern der Rubrik „Ratgebersendung“ zugerechnet. 
- Inwieweit ist die Mediengeschichte bezüglich TV-Sendungen über Gesundheits- und Medizinthemen selber bereits „Medizingeschichte“

Wie schon angeführt, lassen sich in diesem Zusammenhang diese Fragen nicht erschöpfend klären; sie werden jedoch durch die eigentliche Themenstellung aufgeworfen und insofern berührt.

So ist etwa Göpfert (1996), anders als die Autoren hiesiger Statistiken (vgl. Krüger 2002), um eine differenzierte Rubrifizierung der thematischen Breite von Wissenschaftssendungen der BBC bemüht gewesen; er verwendete dabei insgesamt neun Kategorien ${ }^{6}$. Darunter findet sich in der Rubrik „Science in Society “7 die Untergruppe „History of Science“. Das macht im Hinblick auf die zahlreichen wissenschaftshistorischen BBC-Programme, die es im Rahmen eines solchen komparatistischen Untersuchungsansatzes einzuordnen gilt, zweifelsohne Sinn; es impliziert aber auch, dass wissenschaftsgeschichtliche Sendungen im BBC-Programm - und insgesamt auf dem angloamerikanischen Fernsehmarkt (s. u.) - in nennenswerter Quantität produziert und gesendet werden. Insofern gilt es, bereits an dieser Stelle auf die offenkundige Stärke und Qualität britischer Programmformate hinzuweisen. Es handelt sich um die bemerkenswerte Situation, dass bei einem europäischen Fernsehnachbarn eine Angebotsdifferenzierung zu Wissenschaftsthemen vorliegt, die ihresgleichen zumindest bei uns zu suchen scheint. Somit steht mit Göpfert (1996) jedoch nicht nur ein Profil- und Erhebungsinstrument zur Verfügung, das für eine vergleichende Inhaltsbeurteilung taugt. Vielmehr kann seine Untersuchung Impulse, Anregungen und Anstöße für Programmentwicklungen geben und zugleich Maßstäbe für solche Personen bieten, die neue Programme zu beurteilen hätten - seien es Kritiker oder „Gremienkontrolleure“. Die tatsächliche Situation auf dem deutschen Markt wird später noch näher untersucht.

Zunächst gilt es, einer anderen, ebenfalls von Göpfert in derselben Untersuchung geäußerten Annahme zu widersprechen. 1996 noch meinte er: „Science reporting will remain a domain of public broadcasters". Wie wenig er Recht behalten sollte, zeigt bereits der erste Augenschein bei der Betrachtung unserer dualistischen Programmlandschaft nur wenige Jahre später: mit wöchentlichen und täglichen Wissenschaftssendungen bei privaten Fernseh-Veranstaltern mit Vollprogramm-Lizenz ${ }^{8}$. Hinsichtlich der Frage, welche Wissenschaftsinhalte besonders beliebt waren und sind, stellt Göpfert (1996) fest, dass hierbei Naturprogramme quantitativ über lange Zeit in „Führung lagen“, gefolgt jedoch sogleich von Sendungen mit Medizinthemen (Göpfert, 2000; Chang, 2001; Scholz/Göpfert, 1998). Das unterstreicht also das Interesse an diesen Topoi, wobei nähere Untersuchungen das Überwiegen von Ratgeber- und Lebenshilfeaspekten hervorheben (Scholz/Göpfert, 1998; ALM, 2000). Freund und Köck (1994) haben bei der Nachzeichnung der Geschichte der Wissenschaftsberichterstattung im

5 Vgl. z. B. die Hinweise von Essex-Lopresti (1997) in seinem Beitrag über „Medicine and Television" zu Rezipienten- und Fachpresse-Reaktionen auf die erste größere Medizin-Reihe mit 10 Einzelsendungen („Your Life in their Hands") in der BBC.

6 Es ist unverständlich, warum eine solche Differenzierung nicht auch Vorbildfunktion für hiesige Programmstatistiken haben könnte.

7 Es handelt sich um Punkt 7 der Auflistung.

8 Vgl. Pro7 mit „Galileo“ und „Welt der Wunder“, die über ein auffälliges Branding verfügen und (multi-)mediale Wertschöpfung (auch Print) betrieben haben bzw. betreiben; dabei sind sie selber teilweise Glied einer strategischen Wertschöpfungskette eines Teilkonzerns der früheren Kirch-Gruppe gewesen, indem Ausschnittrechte eines Discovery-Sendepaketes (Gesamtausstrahlungsrechte beim ZDF) genutzt wurden. 
bundesrepublikanischen Fernsehen im Zuge der seinerzeit bildungspolitisch eingeforderten Verwissenschaftlichungsoffensive ${ }^{9}$ eine regelrechte Wissenschaftseuphorie im TV ausgemacht. Danach hätten jedoch angewandte Wissenschaftsaspekte im Vordergrund gestanden, lebenspraktische Themen, z. B. in Gestalt von Ratgebersendungen ${ }^{10}$. Diese Feststellung stimmt also mit der Beobachtung Göpferts (2000) überein, wonach die Magazinform zur Berichterstattung und Ratgebung über Fragen der Medizin und Gesundheit damit schon vor der als „Magazinitis“ beschriebenen späteren ersten Hochkonjunktur von Fernsehmagazinen ${ }^{11}$ zur prominenten (und dominierenden) Sendeform in Deutschland erhoben wurde. Bleicher ${ }^{12}$ hat hinsichtlich der dramaturgischen Prinzipien sowie Darstellungs- und Rezipientenaspekten des Magazinformats auf die Verkürzung der Programmpräsentation, das Baukastenprinzip, die Tendenz zur Häppchenkultur und Fragmentierung hingewiesen und unterstrichen, dass Veränderungen in der Narrationsstruktur des Fernsehens auch die Weltwahrnehmung der Zuschauer verändern. Die Feststellung, es werde versucht, ,in Ratgebermagazinen mittels der Langsamkeit eine größere Eindringlichkeit der Information zu erlangen“, ist allerdings - z.T. sendeplatz- und senderabhängig ${ }^{13}$ - dank der auch hier um sich greifenden Dramaturgie der visuellen Narrationsverkürzung und -akzeleration (mit Schnittfrequenzerhöhung, Nutzung digitaler Effekte und Nachbearbeitung ${ }^{14}$ und Einbeziehung akustischer Effekte) vielfach überholt.

Aus dem bisher Festgestellten lassen sich folgende Schlussfolgerungen ziehen:

Zum einen ist die von Krüger (2002) oder von der Arbeitsgemeinschaft der Landesmedienanstalten im Programmbericht (2000) vorgenommene, schon seit langem fortgeschriebene Klassifikation der Inhaltsprofile von Hauptprogrammen hinsichtlich der dort getroffenen Unterscheidung in die Inhaltsklassen „Kultur/Wissenschaft/Technik“, „Soziales/Gesundheit/Arbeitsleben“ und „Tier/Natur/Umwelt“ willkürlich und inkonsistent. Historisch und inhaltlich handelt es sich bei allen um wissenschaftsbezogene Inhalte. Dies mag jedoch nur als Nebenbefund genannt sein. Denn zum anderen fällt die frühe Etablierung der Magazinform im bundesdeutschen TV als dominierende Form der Berichterstattung über Medizin und Gesundheit viel stärker ins Gewicht. Hieraus ergibt sich nämlich im Hinblick auf die eigentliche Themenstellung die Frage, inwiefern sich hiesige TV-Angebote von anderen Märkten, insbesondere dem großen angloameri-

9 Als Reaktion auf Georg Pichts Beschwörung einer Bildungskatastrophe und Ralf Dahrendorfs Abhandlung „Bildung ist Bürgerrecht“ Mitte der sechziger Jahre.

10 Als Prototypen benennen die Autoren hier das im 39. Sendejahr laufende ZDF- „Gesundheitsmagazin Praxis“.

11 In den 70er Jahren.

12 A. a. O., S. $166 f f$.

13 Es bedeutet aus Sicht von Programmentscheidern und Produzenten einen wesentlichen Unterschied, ob es sich um ein Prime-Time-Format mit nationaler Reichweite (z. B. „Praxis - das Gesundheitsmagazin“, ZDF) handelt oder um eine Sendereihe in den so genannten Dritten Programmen. „Die Sprechstunde“, BR, und „Visite“ etwa in N3 berücksichtigen als Magazin überwiegend die zeitgemäße Dramaturgie des Magazins, während „Quivive“ im SFB auch längere Gesprächsstrecken aufweist. Die Entscheidung hierfür wird jedoch meist weniger durch proaktive Redaktionsüberzeugung denn durch schlichten Produktionskostenengpass diktiert; manchmal heißt die Zuschauernachfrage diese „Nolens-volens“-Entscheidung nachträglich gut.

14 Hierbei geht es nicht nur um die durch digitale Effekte angereicherte lineare Verbindung von Bildern; vielmehr werden Bildkompositionen aus z. T. zehn und mehr Bildebenen generiert (z. B. „Picture-in-picture“-Effekte). 
kanischen TV-Markt, unterscheiden, und inwieweit medizinhistorische Topoi im deutschen TV-Angebot im Rahmen der dominierenden Magazine bzw. anderer Programmformen berücksichtigt werden. Tabelle 1 zeigt eine Synopse medizinhistorischer Themen und möglicher - hinsichtlich der inhaltlichen und dramaturgischen Gestaltungsund Vertiefungsmöglichkeiten - wünschenswerter Programmformen. Ein Überblick, wie ihn Tabelle 2 ohne jeglichen Anspruch auf Vollständigkeit erlaubt, lässt erkennen, dass auf dem angloamerikanischen Markt zahlreiche medizin- (und im breiteren Sinne damit wissenschafts-)historische Topoi in programmlicher „Langform“ realisiert und international vertrieben wurden. Eine solche Programmentwicklung ist für den deutschen TV-Markt weitgehend zu verneinen ${ }^{15}$.

Allerdings finden sich Gesundheits-, Medizin-Themen und immer wieder auch integrierte medizinhistorische Aspekte teilweise eklektizistisch in anderen ebenfalls in Deutschland etablierten Formaten, z. B. dokumentarische Lang- und Kurzformen,

\section{Tabelle 1: Medizingeschichte und Programmformen}

\begin{tabular}{|c|c|c|}
\hline $\begin{array}{l}\text { Medizinhistorischer Gegen- } \\
\text { stand / Beispiel }\end{array}$ & $\begin{array}{l}\text { Prädilektive } \\
\text { Programmform }\end{array}$ & $\begin{array}{l}\text { Beispiel } \\
\text { (vgl. Tabelle Nr. 2) }\end{array}$ \\
\hline $\begin{array}{l}\text { Medizinhistorisch bedeutsame } \\
\text { Persönlichkeit }\end{array}$ & $\begin{array}{l}\text { Fernsehdokumentation } \\
\text { Fernsehspiel/Spielfilm }\end{array}$ & $\begin{array}{l}\text { Spielfilm über Sauerbruch } \\
\text { Albert Schweitzer }\end{array}$ \\
\hline $\begin{array}{l}\text { Entdeckungs-/Entwicklungs- } \\
\text { leistung (Diagnostik, Therapie) }\end{array}$ & $\begin{array}{l}\text { Fernsehdokumentation, } \\
\text { auch mit szenischer } \\
\text { Nachstellung }\end{array}$ & $\begin{array}{l}\text { „Cancer Wars“ } \\
\text { (Channel 4) }\end{array}$ \\
\hline $\begin{array}{l}\text { Medizinhistorisches Ereignis } \\
\text { (z. B. Katastrophe, Seuchen) }\end{array}$ & $\begin{array}{l}\text { Fernsehspiel/Spielfilm } \\
\text { (Darstellung des geschicht- } \\
\text { lichen Kontexts am Beispiel } \\
\text { eines Einzelschicksals) } \\
\text { Fernsehdokumentation } \\
\text { Historische Magazinsendung }\end{array}$ & $\begin{array}{l}\text { „Plague Wars“ } \\
\text { (BBC / WGBH) }\end{array}$ \\
\hline $\begin{array}{l}\text { Medizingeschichte im sozial- } \\
\text { und gesellschaftspolitischen } \\
\text { Kontext }\end{array}$ & $\begin{array}{l}\text { Fernsehdokumentation/ } \\
\text { Fernsehspiel } \\
\text { Historische Magazinsendung }\end{array}$ & $\begin{array}{l}\text { „Medizin im Dritten } \\
\text { Reich“ } \\
\text { Zeitgeschichtliche } \\
\text { Dokumentationsreihe } \\
\text { im ZDF, } 2003\end{array}$ \\
\hline
\end{tabular}

Vgl. hierzu Maio (1999, S.127), der hervorhebt, dass es Kennzeichen jeder Fiktion ist, „dass sie aus dem realen Bezugssystem der Wirklichkeit Dinge entnimmt und aus ihnen eine eigene Wirklichkeit formt (...). “ $\mathrm{Zu}$ gleich fragt er: „Wie können Fiktionen Aufschlüsse über historische Zusammenhänge geben?“; in diesem Kontext äußert er, dass der Film (zwar) auch Realität schaffe, zugleich aber auch Realität widerspiegele. Insofern handelt es sich m. E. um eine interessante und zudem zuschauerattraktive Möglichkeit der TV-Narration.

** Auf die Bedenken von Hattendorf (1999, S. 59) zur nichtfilmischen und filmischen Realität im Dokumentarfilm wird ausdrücklich verwiesen.

15 Es sei jedoch auf Filmangebote außerhalb des TV-Marktes verwiesen, z. B. auf Dokumentationen, aber auch szenische Nachstellungen (z. B. über die Entdeckung der Tuberkulose-Erreger durch Robert Koch), die beim IWF (Institut für den Wiss. Film, heute IWF Wissen und Medien $\mathrm{gGmbH}$, Göttingen) erschienen und insbesondere für den Einsatz im wissenschaftlichen Bereich herangezogen wurden. 
Sachfilme, Talksendungen, Livesendungen sowie Magazine unterschiedlicher Zusammensetzung und Intentionalität.

Unabweisbar ist die Frage zu stellen, warum es im deutschen Fernsehen kein dem angloamerikanischen TV-Markt vergleichbares Programmangebot gibt, und wie überhaupt medizingeschichtliche Aspekte Erwähnung finden. Wie dargestellt, kam es im hiesigen öffentlich-rechtlichen Programm schon frühzeitig zur Konzentration auf die Magazinform bei Medizin- und Gesundheitsthemen. Dieses bedingte eine Konzentration in Darstellungslänge und -tiefe, häufig eine Mehrthemen-Mischung und einen begrenzten und dann meist integrierend gehandhabten Rückgriff auf Medizinhistorie. So wurden selbstverständlich Geschichte (und manchmal auch Geschichten) um eine Entdeckung oder Verfahrensentwicklung, Persönlichkeiten in Verbindung hiermit und politischer wie gesellschaftlicher Kontext berücksichtigt - meist jedoch zwangsläufig verknappt als Rückblende integriert oder historisch-linear nacherzählt im Rahmen der jeweiligen Kurzbeiträge. Ausnahmen können diese Üblichkeit nur bestätigen. Über fiktionale Einzelrealisierungen hinaus (s. Tabelle 2) gehören hierzu Dokumentationen aus Anlass von Jahrestagen, wie z. B. die ZDF-Dokumentation „100 Jahre Röntgen - 100 Jahre Durchblick"16. Hinzu kommen fernsehadaptierte Medialisierungen medizingeschichtlicher Phänomene. Diese lassen sich z. T. auch unmittelbar an noch lebenden Persönlichkeiten oder Zeitzeugen festmachen. Als Beispiel mag die Live-Übertragung einer Bypass-Operation aus der Fundación Favaloro in Buenos Aires dienen, die dort der „Vater“ dieser Technik, Dr. René Favaloro, über 30 Jahre nach der Etablierung als Methode durch ihn in Cleveland persönlich durchführte. Dieser Sendung im Rahmen der ZDF-Reihe „Praxis - das Gesundheitsmagazin“ am 8. April 1998 schloss sich am späten Abend eine mehrstündige „Herz-Nacht aus Buenos Aires“ als „Praxis extra“-Produktion mit zahlreichen Live-Operationen und -Interventionen auf dem avanciertesten Stand der Herzchirurgie unter Hinzuziehung zweier Herzchirurgen aus Deutschland für die ergänzende Fachkommentierung an, die streckenweise auch noch nachts bis weit über eine Million Zuschauer verfolgten (Idee, Moderation ${ }^{17}$ und Gesamtleitung: Christian Floto). Diese Produktion medialisierte unmittelbar eine bedeutende, wissenschaftlich relevante Technik- und Therapieentwicklung durch Personalisierung, Aktualisierung und strategische Aufmerksamkeitsökonomie-Steigerung ${ }^{18}$ mit dem Mittel der Live-Darstellung ${ }^{19}$, das zudem einem weiteren Authentizitätsgewinn im Interesse der Attribuierung an die Gesamtreihe „Praxis“ dienen sollte.

16 ZDF in Koproduktion mit SVT, 1995, 45 Minuten Länge.

17 Zusammen mit Dr. Dierk Heimann.

18 Allerdings über die von Hickethier (1999, S. 96) angemerkte Tendenz der FernsehprogrammEntwicklung zur gezielten Erzeugung von Medienereignissen hinaus: als zeitgeschichtlich begründetes, in Form, Dramaturgie und Darstellungsmitteln herausgehobenes, virulentes Fernsehereignis mit anzustrebender Nachhaltigkeit (Anspruch z. B.: „Daran erinnert sich ein $\mathrm{Zu}-$ schauer auch noch viele Jahre später.").

19 Hierzu sei auf die von Hoffmann (2000) angeführte Diskussion der „Live-Philosophie“ verwiesen, wobei festzustellen ist, dass für den Medizinbereich die Live-Darstellung von Abläufen im Operationssaal in Verbindung mit der kommentierten und durch erklärende Einspielbeiträge ergänzten Darstellung von Operationstechniken, die fernsehgeschichtlich durch die ZDFReihe „Praxis - das Gesundheitsmagazin“ als Regelangebot etabliert wurde, andere Erfahrungen und Vorgehensweisen gelten, als sie dort geäußert werden. So ist z. B. die Auffassung unzutreffend, Live-Sendungen seien im Allgemeinen durch und durch inszeniert. 
Tabelle 2: Beispiele für TV-Produktionen mit medizinhistorischem Content

\begin{tabular}{|c|c|}
\hline \multicolumn{2}{|c|}{ 1. Medizinhistorische (teil-)fiktionale Angebote } \\
\hline 1.1. $T V$ & \\
\hline Serien & Microbes and Men (18.9. - 23.10.1974). UK: BBC \\
\hline Einzelsendungen & $\begin{array}{l}\text { Entebbe Encounter and The sleep of Death (1989). } \\
\text { UK: Wellcome Trust } \\
\text { Hospital, } 1922 \text { (1972). UK: BBC } \\
\text { Hair Soup (1992). UK: Yorkshire TV } \\
\text { And the Band Played On (1993). USA: HBO } \\
\text { Against All Odds (1994). UK: BBC }\end{array}$ \\
\hline \multicolumn{2}{|l|}{ 1.2. Kino } \\
\hline Sozialgeschichte der Medizin & $\begin{array}{l}\text { Die Ehe des Dr. med. Dannwitz (1956). BRD } \\
\text { One flew over the Cuckoo's Nest (1975). USA } \\
\text { The Aboriginal (1963). AUS } \\
\text { Awakenings (1990). USA } \\
\text { Girl, Interrupted (1999). USA }\end{array}$ \\
\hline Biografien von Entdeckern/ Medizinern & $\begin{array}{l}\text { Paracelsus (1943). BRD } \\
\text { Sauerbruch - Das war mein Leben (1945). BRD } \\
\text { Lorenzo's Oil (1992). USA } \\
\text { Mesmer (1994). USA } \\
\text { Patch Adams (1998). USA }\end{array}$ \\
\hline \multicolumn{2}{|c|}{ 2. Nonfiktionale medizinhistorische Einzelproduktionen } \\
\hline Koproduktionen & $\begin{array}{l}\text { The Keys of Paradise (1979). USA: BBC/WGBH } \\
\text { Invisible Enemies (1992). UK: Channel 4/AUS: Film } \\
\text { Australia } \\
\text { White Heat (1994). USA: BBC/Discovery Channel } \\
\text { Pandemic (1999). Associated Producer Inc. Films for } \\
\text { BBC/WGBH/CBC/NRK } \\
\text { Plague Wars (1998). USA: BBC/WGBH } \\
\text { The Plague Doctors (1996). } \\
\text { CBC/NOVA/WGBH/Channel } 4 \\
\text { The People's Century: 1954 - Living Longer (1997). } \\
\text { USA: BBC/WGBH }\end{array}$ \\
\hline Deutschland & $\begin{array}{l}100 \text { Jabre Röntgen - } 100 \text { Jabre Durchblick (1995). } \\
\text { Deutschland: ZDF/SVT }\end{array}$ \\
\hline UK & $\begin{array}{l}\text { Florence Nightingale (1978). UK: BBC } \\
\text { The Courage to Fail (1987). UK: BBC } \\
\text { Darwin, the Devil's Chaplain (1991). UK: BBC } \\
\text { Secrets of Porton Down (1994). UK: Carlton } \\
\text { Television } \\
\text { Linus Pauling (1994). UK: BBC } \\
\text { Albert Schweitzer - Darkness E Light (1994). UK: } \\
\text { BBC } \\
\text { Prisoners of the Forgotten Plague (1998). UK: BBC }\end{array}$ \\
\hline
\end{tabular}


UK (Fortsetzung)

The Three Valleys of St. Lucia (1984). UK: Channel 4 The Kuru Mystery. (1984) UK: Channel 4 Stories from an African Hospital (1991). UK: Channel 4 Out of Sight (1992). UK: Channel 4 The Plague (1993). UK: Channel 4 Darwin (1994). UK: Channel 4

The Lynchburg Story (1994). UK: Channel 4 Mary Stopes (1995). UK: Channel 4 The Ladykillers (1995). UK: Channel 4 The Saga of Life (1996). UK: Channel 4 A Woman's Fight to Choose (1997). UK: Channel 4 The Infirmary (1998). UK: Channel 4 Shell Shock (1998). UK: Channel 4 Cancer Wars (1998). UK: Channel 4 The Babymakers (1999). UK: Channel 4 Three Thin Ladies (2000). UK: Channel 4

USA In the Shadow of the Reich: Nazi Medicine (1997). USA: Michalczyk, John

Inventions that Changed Our Lives - Medicine (2000). USA: Goldhill Home Media The People's Plague (1995). USA: PBS Brain Transplant (1992). USA: NOVA Ebola - The Plague Fighters (1996). USA: NOVA Cut to the Heart (1997). USA: NOVA The Brain Eater (1998). USA: NOVA Electric Heart (1999). USA: NOVA Cracking the Code of Life (2001). USA: NOVA Cancer Warrior (2001). USA: NOVA Dr. Folkman's War (2001). USA: NOVA Young Dr. Freud (2002). USA: NOVA War on Cancer (1996). USA: Discovery Aspirin. A Bitter Pill (2001). USA: Discovery

Canada Something Hidden - A Portrait of Wilder Penfield (1979). CAN: CBC

\section{Sender, Reihen, Serien, Magazine}

3.1. Formate mit medizinhistorischen Anteilen (inkl. Beispielen)

Deutschland

- 3sat

Nano 100 Jabre Krebsforschung (18.2.2000) Albert Schatz - der Mann, der die Tuberkulose besiegte (10.12.2001)

- Arte Hippokrates

UK

- $\mathrm{BBC}$

Superbuman

Reputations

Timewatch 
UK (Fortsetzung)

- Channel 4
Great Britons

Secret History

Secret Lives

Equinox

The Pulse

Witness

Disabling World

Dispatches

\section{USA}

- Discovery Channel

Medical Mysteries

3.2. Sender mit Doku-Slots, die teilweise mit medizinhistorischen Themen besetzt werden (teilweise inkl. Beispielen):

\section{Deutschland}

- Arte

- 3sat

- Phoenix

Homo Xerox - Ich lasse mich klonen. (25. 05. 2001, 13.30 Uhr). urspr.: (1999). D: ZDF.

Mensch und Mikrobe - Die Suche nach den Impfstoffen. (2tlg.). (26. 06. 2001, 19.15 Uhr). urspr.: D: ZDF.

Tote sagen aus - Die Geschichte der Gerichtsmedizin. (2tlg.). (07. 12. 2001, 8.15 Uhr). urspr.: (1999). D: ZDF/ Arte.

- XXP Abentener Medizin. (3tlg. Spiegel-TV Dokumentation).

Supermensch - die Heilkraft des Körpers. (6tlg. BBCDokumentation).

Günter Gaus im Gespräch mit Moritz Mebel. (dctp Interview).

Die Babymacher - von der künstlichen Befruchtung zum Designerkind. (13.11. 2002, 16.55 Uhr). (Spiegel TV Dokumentation).

- RTL \& Sat.1 Spiegel-TV

$\mathrm{UK}$

- BBC Omnibus

USA

- PBS

- Discovery Channel

- "The Learning Channel” (Discovery)

- WGBH

3.3. Die wichtigsten TV-Contentproduzenten bzgl. Medizin-Dokumentationen

Deutschland Spiegel TV (Content-Übernahme aus internationalen Produktionen)

Discovery Deutschland (Content-Übernahme aus internationalen (Ko-) Produktionen) 


\begin{tabular}{ll} 
UK & BBC \\
& Channel 4 \\
\hline USA & Discovery \\
& WGBH \\
& $P B S$ \\
& NOVA \\
& $A M E X$ \\
\hline Canada & $C B C$ \\
\hline
\end{tabular}

Dieses Beispiel darf jedoch nicht darüber hinweg täuschen, dass es sich hierbei im deutschen Fernsehprogramm um Einzelerscheinungen handelt. Mehrteilige medizinhistorische Produktionen, wie etwa aus derselben Fachdisziplin die Geschichte der Herztransplantation als BBC-Eigenproduktion (s. Tabelle 2), entbehren einer Entsprechung in der deutschen Fernsehproduktions- und Programmplanungsgeschichte. Immerhin sind in angloamerikanischen Dokumentationen auch Einzelbeobachtungen im Kontext aktueller wissenschaftlicher Experimente ausführlich thematisierbar, die therapeutische Horizonte eröffnen könnten, ohne dass diese bereits geklärt wären - Themen also mit allenfalls prospektiver medizinhistorischer Potenz. ${ }^{20}$

$\mathrm{Zu}$ fragen ist nach den Gründen der sich auch aus den in der Tabelle 2 aufgeführten Produktionsbeispielen ergebenden Phänomene. Warum ist deutsche Produktions- und Programmplanung so offensichtlich anders ausgerichtet, was (explizite) medizinhistorische Sendungen anbelangt?

Einen wesentlichen Aspekt machen sicherlich die unterschiedliche Bedeutung von Dokumentationen und deren Wertigkeit in der Zuschauerrezeption aus. Im BBC-Programm gibt es mehrere thematisch weitestgehend offene Sendeslots für Dokumentationen, in denen auch Medizin und Medizingeschichte, sogar über mehrere Folgen hinweg, thematisiert werden. Dem stehen im (öffentlich-rechtlichen) deutschen Programm-Angebot zwar in Sendezeit und Produktionsaufwand ebenfalls hochwertige Möglichkeiten $^{21}$ gegenüber; diese werden jedoch bezüglich der Themenfarbe und -breite konkreter und enger besetzt.

20 In der am 12.5.2002 im BBC-Slot von VOX unter dem deutschen Titel „Ecstasy als Heilmittel“ ausgestrahlten BBC-Produktion drehte es sich beispielsweise um die zufällige Anwendungsbeobachtung bei Tim, einem 39-jährigen Parkinson-Patienten, der unter missbräuchlicher Anwendung von Ecstasy einen völligen Rückgang der Beschwerden, insbesondere der dyskinetischen Symptomatik, beobachtete. Die 50-minütige Dokumentation beschreibt u. a. die daraufhin am Londoner Hammersmith-Hospital bei ihm unternommenen Einzeltests. Die dramaturgische Gestaltung sowie die Personalisierung kulminieren in der Erkenntnis eines Forschungsbedarfs mit enormem Heilungspotenzial. Beim Rezipienten wird zudem das Gefühl geweckt, quasi bei der "Geburtsstunde“ einer unerhört wichtigen Entdeckung dabei gewesen zu sein. Für diese Qualität von Programmentscheidung und -begründung gibt es in Deutschland keine Tradition. Die Kontributionen der ZDF-Sendereihe "Gesundheitsmagazin Praxis“ insbesondere im Zusammenhang mit herausgehobenen aktuellen Entwicklungen (z. B. Operationen unter Akupunktur, Herztransplantation, HIV-Forschung) haben trotz redaktioneller Ambitioniertheit keinen Paradigmenwechsel in Programmplanung und -kultur, insbesondere auch nicht bei konkurrierenden Programmveranstaltern, kenntlich werden lassen.

21 Vgl. z. B. den 19.30-Uhr-Termin im ZDF-Programm am Sonntag. 
Zudem entwickelte sich auf dem Boden der Grundspannung einer Wechselbeziehung zwischen Programmtradition, „gelernter Rezeption“ und heutigen Programmplanungskriterien eine sich gegenseitig bedingende Zwangsläufigkeit, die kommunikative (Abstimmungs- und Argumentationsprozesse zwischen Redaktionen, Programmplanung und strategischer Leitungsebene, also Programmdirektion bzw. Intendanz) wie rezeptionsgeschichtliche Aspekte 22 aufweist.

Vor diesem Hintergrund ist die Titelfrage dieses Beitrags zu beantworten: Es handelt sich offensichtlich im deutschen Fernsehen um ein Dosisproblem. Unbestritten und üblich sind und bleiben die medizinhistorischen Rückgriffe im Zuge von Magazinbeiträgen (z. B. über therapeutische und diagnostische Verfahren). Hier gehört es zum narrativen Standard, auch die historische Perspektive „einzubauen“. Leider gerät die dramaturgische Realisierung dabei vielfach recht einfallsarm, da Autor/Produzent sie eher als „Pflichtpetitesse“ betrachten, derer es sich zu entledigen gilt. Im Rahmen eines Magazinbeitrags verhelfen darüber hinaus die Längenbegrenzung sowie der Mangel an Bildmaterial $^{23}$ und damit an adäquater Medialisierbarkeit unter heutigen Rezeptionsgewohnheiten zu einer hinreichenden Argumentationsbasis bei diesbezüglichen Rückfragen und Anmerkungen aus Redaktions- und Rezipientenkreisen. Somit hat ein gewisses $\mathrm{Maß}$ an dramaturgisch-gestalterischer Kreativitäts- und Lieblosigkeit im Zusammenwirken mit einer zunehmenden gesellschaftlichen Wertschöpfungsorientierung dazu geführt, dass die Betrachtung geschichtlicher Zusammenhänge bei Medizinthemen vernachlässigt wird, und zudem durch anthropozentrische Fixierung gekennzeichnet ist bzw. sich zunehmend in einer kasuistisch-personalisierenden Narrationsstruktur („Das ist Josef K.; seit zwei Jahren leidet er an ...") ergeht, wenn nicht sogar erschöpft.

So gesellt sich zu dem Dosis- auch ein Darreichungsproblem: Neben den Bilderhunger, dem insgesamt bei der fernsehgerechten Umsetzung von historischen Themen mangels Material häufig schwer zu genügen ist, tritt das Erfordernis zeitgemäßer Fernsehnarration und -dramaturgie, die z. T. aufgrund ihres Inszenierungs- oder Postproduktionsaufwands einen verhältnismäßig hohen Produktionsetat erfordern. ${ }^{24} \mathrm{Bei} \mathrm{Be}-$ achtung programmwirtschaftlicher Entscheidungskriterien (Sendeminutenpreis nach Programmkategorie, Wiederverwertbarkeit, Veräußerbarkeit etc.) sind die Realisierungsgrenzen meist schnell erreicht. Nur durch Koproduktion (Problem hier: Aufwand, Zeit bis Vertragsschluss; diese kann der Auftragsproduzent meist nicht auch noch organisieren) oder erkennbare Ankaufsoptionen durch andere Broadcaster lassen sich diese Kostenlimits überschreiten. Dem steht einschränkend die besondere deutsche Dramaturgie entgegen, die sich formal in dem international unüblichen Format „42 Minuten“ für eine „Lang-Dokumentation“ ausdrückt. International üblich sind 50-55 Minuten (s. o.), und hierfür stehen natürlich bei angloamerikanischen Broadcastern die entsprechenden Slots zur Verfügung. Das bedeutet für den Fall der internationalen Vermarktbarkeit das Erfordernis, eine entsprechende „internationale Version“ zu erstellen, die nicht einfach nur 10 Minuten länger ist, sondern auch eine veränderte Dramaturgie (z. B. eine üblicherweise längere Exposition) unabdingbar einfordert. Insofern hat die

22 Der Wandel des Fernsehens von einem Angebots- zu einem Nachfragemedium trägt zur Verfestigung derartiger Entwicklungen bei.

23 Vgl. den von Seeßlen (1997) verwendeten Begriff vom „Bilderhunger des wuchernden Mediums".

24 Im Vergleich zum durchschnittlichen Sendeminutenpreis anderer nonfiktionaler Produktionen; fiktionale Programme kosten üblicherweise ein Mehrfaches davon. 
(nachfrageorientierte) Bedienung deutscher Rezipienten-Interessen in Darreichung und Dosierung zur Spezifität und weitgehenden internationalen Markt-Inkompatibilität deutscher TV-Formate zu Medizin- und Medizingeschichte-Themen geführt.

Ist eine Lösung oder Weiterentwicklung dieses Problems denkbar? Das deutsche TVProgramm wird für eigene medizinhistorische Dokumentationen auch künftig keine Sendeplätze ausweisen, da bestehende Programmslots in ihrem thematischen Spektrum wohl nicht erweitert werden und programmplanerisch eher die Versiegelung eines früher heterogenen Programm-Spielplanes durch Wöchentlich- oder Täglichstellung kostengünstiger Formate (z. B. Daily Talks wie „Kerner“ oder „Beckmann“) zu beobachten ist. ${ }^{25}$ Dies führt zu einer weiteren Versteppung der programmlichen Möglichkeiten in den so genannten Hauptprogrammen öffentlich-rechtlicher Anbieter. Die Spartenkanäle bieten theoretisch zwar hinreichend Spielfläche, auch für medizinhistorische Themen - gleichzeitig ist deren Spielraum für Eigenproduktionen aufgrund sehr beschränkter Geldmittel außerordentlich gering; insofern kommen sie als Auftraggeber/Produzenten kaum in Betracht. ${ }^{26}$

Die Öffnung und Erweiterung des Programmangebots wird sich durch Internationalisierung und Globalisierung weiter verstärken. Hierzu dienen die bestehenden Allianzen mit international agierenden Content-Imperien, die sowohl zu Regel-Slots bei öffentlich-rechtlichen Anbietern (vgl. „Discovery“, ZDF, fünfmal pro Woche im Nachmittagsprogramm) als auch zu „Fenstern“ bei privaten Fernsehveranstaltern geführt haben. Der BBC-Slot bei VOX ist hierfür das beste Beispiel; „Spiegel TV“ greift ebenfalls auf BBC-Programme zurück, bearbeitet sie jedoch vielfach, verfügt insofern meist über Ausschnittrechte, die die BBC üblicherweise erst einige Zeit nach der britischen ErstAusstrahlung veräußert. ${ }^{27}$ Über diese Allianzen und Programm-Pakete, mit denen zum Zwecke der Mehrfachverwertung künftig auch (weitere, digitale) Spartenkanäle bestückt werden dürften, gelangt zunehmend auch medizinhistorischer TV-Content nach Deutschland. So regelt sich der Programmwirtschafts- und Programmplanungsmarkt nach den Kriterien „Internationalität“ und „Mittelknappheit“ zunehmend selber; die Versiegelung von Programmslots durch das Einziehen täglicher Programmschienen (s. o.) tut ein Übriges. Für wirklich originäre Planungs- und Gestaltungsaufgaben und -bedarf verbleibt zunehmend nur noch der Prime Time-Bereich. Und auch hier wird der eigenständig gestaltende Spielraum durch Outsourcing-Produktionsverträge und „globalisierte Formate“ enger. Doch in punkto medizinhistorischer Content wird sich die Frage nach Dosis- oder Darreichungsproblemen zunehmend relativieren - der Programm-Globalisierung sei Dank ...!

\section{Literatur}

Arbeitsgemeinschaft der Landesmedienanstalten ALM (Hrsg.) (2000): Programmbericht zur Lage und Entwicklung des Fernsehens in Deutschland 2000/2001. Konstanz: UVK.

Bleicher, J. K. (1999): Fernsehen als Mythos - Poetik eines narrativen Erkenntnissystems. Opladen/Wiesbaden: Westdeutscher Verlag.

25 Vgl. die von Hickethier (1999, S. 89ff.) beschriebenen „Trends der Programmentwicklung im öffentlichen und privaten Fernsehen“.

26 Arte mag hierbei wegen höherer Geldmittel als Ausnahme gesehen werden.

27 Das ist insofern wichtig, als dass bei der Verwendung von Ausschnitten meist nur auf ältere Produktionen zurückgegriffen wird. 
Chang, Y-C. (2001): Gesundheit in Fernsehen und Internet. Dissertation. Sozialwiss. Fakultät der Georg-August-Universität zu Göttingen.

Essex-Lopresti, Michael (1997): Medicine and Television. Journal of Audiovisual Media in Medicine, 20, 2, S. 61-64.

Freund, B.; Köck, W. K. (1994): Wissenschaftsvermittlung durch Fernsehen zwischen Information und Unterhaltung. In: Ludes, P.; Schumacher, H.; Zimmermann, P. (Hrsg.): Geschichte des Fernsebens in der Bundesrepublik Deutschland, Bd. 3, München, S. 175-201.

Göpfert, W. (1996): Scheduled Science: TV Coverage of science, technology, medicine and social science and programming policies in Britain and Germany. Public Understand. Soc. 5, S. $36-$ 374.

Göpfert, W. (2000): Gängige Themen: Medizin und Gesundheit. In: Stadelhofer, C.; Körting, G. (Hrsg.): Seniorstudierende online. Mössingen-Talheim: Talheimer, S. 205-213.

Hattendorf, M. (1999): Dokumentarfilm und Authentizität. Ästhetik und Pragmatik einer Gattung. Konstanz.

Hickethier, K. (1999): Trends in der Programmentwicklung im öffentlich-rechtlichen und im privaten Fernsehen. In: Schröder, H.-D. (Hrsg.), Entwicklung und Perspektiven der Programmindustrie. Baden-Baden: Nomos (Symposien des Hans-Bredow-Instituts; 17), S. 89 - 103.

Hoffmann, K. (2000): Wie „lebendig“ kann Fernsehen sein? In: Hallenberg, G.; Schanze, H. (Hrsg.): Live is Life - Mediale Inszenierungen des Authentischen. Nomos Verlagsgesellschaft, Baden-Baden, S. 163 - 176.

Krüger, U. M. (2002): Inhaltsprofile öffentlich-rechtlicher und privater Hauptprogramme im Vergleich. Media Perspektiven 10, S. 512 - 532.

Leiser, E. (1992): Legitime Manipulation. Dokumentarfilm und Geschichte am Beispiel der NSVergangenheit. Medium 2, S. 25.

Maio, G. (1999): Krankheit und Medizin in der Geschichte des Films. Dargestellt am Beispiel der Epilepsie. Die Psychotherapentin 10, S. $126-142$.

Scholz, E.; Göpfert, W. (1998): Wissenschaft und Fernsehen. Eine Vergleichsstudie 1992 - 1997. Manuskript, Institut für Publizistik und Kommunikationswissenschaften der FU Berlin.

Schröder, H.-D. (Hrsg.) (1999): Entwicklung und Perspektiven der Programmindustrie. Baden-Baden: Nomos (Symposien des Hans-Bredow-Instituts; 17).

Seeßlen, G. (1997): Kinderschänder, Bildfälscher. Konkret 2, S. 46. 